\title{
Applications of Mann's method to the split common fixed point problem
}

\author{
Youli Yua , Zhangsong Yaob, Yaqin Wang ${ }^{\mathrm{c}, *}$, Xiaoli Fang ${ }^{\mathrm{c}}$ \\ ${ }^{a}$ School of Mathematics and Information Engineering, Taizhou University, Linhai 317000, China. \\ ${ }^{b}$ School of Information Engineering, Nanjing Xiaozhuang University, Nanjing 211171, China. \\ ${ }^{c}$ Department of Mathematics, Shaoxing University, Shaoxing 312000, China.
}

Communicated by Y. H. Yao

\begin{abstract}
In the present paper, we suggest a new fixed point method for solving the split common fixed point problem of directed operators. We present an iterative algorithm based on Mann's method. We prove that the presented algorithm converges weakly to a solution of the split common fixed point problem of directed operators. (C)2017 All rights reserved.
\end{abstract}

Keywords: Split common fixed point problem, directed operator, weak convergence.

2010 MSC: 47H10, 65J15.

\section{Introduction}

Many problems in nonlinear analysis can be reformulated as a problem of finding a fixed point of a nonlinear operator. Iterative methods for finding fixed points of nonlinear operators have received vast investigation: Browder et al. [3], Mainge [11], Nakajo et al. [13], Shioji et al. [15], Xu [20], and Yao et al. [24, 25]. An important way for finding fixed points of nonlinear operators is Mann's method [19] which generates a sequence $\left\{x^{k}\right\}$ by the form

$$
\text { (Mann's method:) } \quad x^{k+1}=\left(1-\alpha_{k}\right) x^{k}+\alpha_{k} \mathcal{T} x^{k}, k \geqslant 0,
$$

where $\left\{\alpha_{k}\right\}_{k \geqslant 0} \subset(0,1)$ and $\mathcal{T}$ is a nonlinear operator.

Mann's method and its variant form have been studied extensively. Especially, in [10, 16, 22], modified Mann's has been introduced and studied. The aim of the present article is to apply Mann's method for solving the split common fixed point problem. To begin with, let us recall some related notations and existing results in the literature.

Throughout, assume that $\mathcal{H}_{1}$ and $\mathcal{H}_{2}$ are two real Hilbert spaces, $\langle\cdot, \cdot\rangle$ denotes the associated scalar product and $\|\cdot\|$ stands for the corresponding norm. Let $\mathcal{U}: \mathcal{H}_{1} \rightarrow \mathcal{H}_{1}$ and $\mathcal{V}: \mathcal{H}_{2} \rightarrow \mathcal{H}_{2}$ be two nonlinear

\footnotetext{
${ }^{*}$ Corresponding author

Email addresses: yuyouli@tzc.edu.cn (Youli Yu), yaozhsong@163.com (Zhangsong Yao), wangyaqin0579@126.com (Yaqin Wang), fx10418@126.com (Xiaoli Fang)
}

doi:10.22436/jnsa.010.03.27 
operators. Denote the fixed point sets of $\mathcal{U}$ and $\mathcal{V}$ by $\operatorname{Fix}(\mathcal{U})$ and $\operatorname{Fix}(\mathcal{V})$, respectively. Let $\mathcal{A}: \mathcal{H}_{1} \rightarrow \mathcal{H}_{2}$ be a bounded linear operator with its adjoint $\mathcal{A}^{*}$.

Recall that the split common fixed point problem is to find an element $u^{\sharp} \in \mathcal{H}_{1}$ satisfying

$$
\text { (SCFPP:) } \quad u^{\sharp} \in \operatorname{Fix}(\mathcal{U}) \text { and } \mathcal{A} u^{\sharp} \in \operatorname{Fix}(\mathcal{V}) \text {, }
$$

which was firstly introduced by Censor and Segal in [6].

An important special case: If $\mathcal{U}$ and $\mathcal{V}$ are the projections, then SCFPP (1.2) reduces to the split feasibility problem, which is to find a point $u^{\sharp}$ such that

$$
\text { (SFP:) } \quad u^{\sharp} \in \mathcal{C} \text { and } \mathcal{A} u^{\sharp} \in \mathcal{Q} \text {, }
$$

where $\mathcal{C} \subset \mathcal{H}_{1}$ and $\mathcal{Q} \subset \mathcal{H}_{2}$ are two nonempty closed convex sets.

Such problems arise in the field of intensity-modulated radiation therapy when one attempts to describe physical dose constraints and equivalent uniform dose constraints within a single model ([5]). Some efforts are being extended to solve the split feasibility problem: Byne [4], Wang and Xu [18], and Xu [21].

Note that SCFPP (1.2) can be translated to solve a fixed point problem. In fact, SCFFP (1.2) equals the following fixed point equation

$$
\text { (FPE:) } \quad \chi^{\sharp}=U\left(\chi^{\sharp}-\mu \mathcal{A}^{*}(\mathcal{J}-\mathcal{V}) \mathcal{A} \chi^{\sharp}\right),
$$

where $\mu>0$ is a constant.

By using FPE (1.4), some iterative algorithms have been presented for solving SCFPP (1.2), see, e.g., Chang et al. [7], He and Du [9], Moudafi [12]. Very recently, Wang [17] presented the following iterative algorithm which is generated by the following iteration

$$
x^{k+1}=x^{k}-\mu\left[\left(x^{k}-U\left(x^{k}\right)+\mathcal{A}^{*}(\mathcal{J}-\mathcal{V}) \mathcal{A} x^{k}\right], k \geqslant 0,\right.
$$

where the stepsize $\mu$ is in the interval $\left(0, \frac{1}{\max \left\{1,\|\mathcal{A}\|^{2}\right\}}\right)$ and $\mathcal{U}$ and $\mathcal{V}$ are directed operators. They proved the weak convergence of the sequence $\left\{x^{k}\right\}$ to the solution of SCFPP (1.2).

On the other hand, Yao et al. [23] introduced the following fixed point equation to solve SCFPP (1.2)

$$
\chi^{\sharp}=\mathcal{U} x^{\sharp}-\mu \mathcal{A}^{*}(\mathcal{J}-\mathcal{V}) \mathcal{A} x^{\sharp},
$$

where $\mu>0$ is a constant and $\mathcal{U}$ and $T$ are directed operators.

It is our main purpose in this paper that we extend fixed point equation (1.5) to a general form with variant parameters. Subsequently, we apply fixed point equation (1.5) and Mann's method (1.1) for solving the split common fixed point problem (1.2). Weak convergence theorem is given under some mild assumptions.

\section{Preliminaries}

Let $\mathcal{H}$ be a real Hilbert space, and $\mathcal{C} \subset \mathcal{H}$ a nonempty closed convex set. Recall that an operator $\mathcal{U}: \mathcal{C} \rightarrow \mathcal{C}$ is said to be directed if

$$
\left\|\mathcal{U} x-x^{\sharp}\right\|^{2} \leqslant\left\|x-x^{\sharp}\right\|^{2}-\|\mathcal{U} x-x\|^{2},
$$

or equivalently,

$$
\|x-\mathcal{U} x\|^{2} \leqslant\left\langle x-\mathcal{U} x, x-x^{\sharp}\right\rangle,
$$

for all $x \in \mathcal{C}$ and $x^{\sharp} \in \operatorname{Fix}(\mathcal{U})$.

The class of directed operators was introduced and investigated by Bauschke and Combettes in [2] and by Combettes in [8]. The class of directed operators is an important class since it includes the orthogonal projections and the subgradient projectors which are fundamental in the convex optimization 
[1] and it allows a complete characterization of Fejér-monotonicity. Recall that a sequence $\left\{x^{k}\right\}$ is called Fejér-monotone with respect to a given nonempty set $\Omega$ if for every $u \in \Omega$,

$$
\left\|x^{k+1}-u\right\| \leqslant\left\|x^{k}-u\right\| \text { for all } k \geqslant 0 .
$$

An operator $\mathcal{U}$ is said to be demiclosed, if for any sequence $x^{k}$ which weakly converges to $\tilde{x}$, and if the sequence $U\left(x^{k}\right)$ strongly converges to $z$, then $U(\tilde{x})=z$.

In any Hilbert space $\mathcal{H}$, the following conclusion holds:

$$
\|x+y\|^{2}=\|x\|^{2}+2\langle x, y\rangle+\|y\|^{2},
$$

for all $x, y \in \mathcal{H}$.

Next we adopt the following notation:

- $x_{n} \rightarrow x$ means that $x_{n}$ converges weakly to $x$;

- $\omega_{w}\left(x_{n}\right):=\left\{x: \exists x_{n_{j}} \rightarrow x\right\}$ is the weak $\omega$-limit set of the sequence $\left\{x_{n}\right\}$.

Lemma 2.1 ([14]). Let $\mathcal{H}$ be a Hilbert space and $\left\{x^{k}\right\}$ a sequence in $\mathcal{H}$ such that there exists a nonempty set $\Omega \subset \mathcal{H}$ satisfying the followings:

(i) For every $y \in \Omega, \lim _{k \rightarrow \infty}\left\|x^{k}-y\right\|$ exists.

(ii) Any weak-cluster point of the sequence $\left\{x^{k}\right\}$ belongs to $\Omega$.

Then, there exists $\bar{x} \in \Omega$ such that $\left\{x^{k}\right\}$ weakly converges to $\bar{x}$.

\section{Main results}

In this section, we will focus on SCFPP (1.2). Let $\mathcal{H}_{1}$ and $\mathcal{H}_{2}$ be two real Hilbert spaces. Let $\mathcal{U}$ : $\mathcal{H}_{1} \rightarrow \mathcal{H}_{1}$ and $\mathcal{V}: \mathcal{H}_{2} \rightarrow \mathcal{H}_{2}$ be two directed operators. Let $\mathcal{A}: \mathcal{H}_{1} \rightarrow \mathcal{H}_{2}$ be a bounded linear operator with its adjoint operator $\mathcal{A}^{*}$. Assume that SCFPP (1.2) is consistent, i.e., its solution set, denoted by $\Omega$, is nonempty.

First, we prove that SCFPP (1.2) is equivalent to a new fixed point equation which plays an important role for constructing our algorithm.

Lemma 3.1. $u^{\sharp} \in \Omega$ if and only if $u^{\sharp} \in \operatorname{Fix}\left(\mathcal{U}-\mu_{k} \mathcal{A}^{*}(\mathcal{J}-\mathcal{V}) \mathcal{A}\right)$ where $\mu_{\mathrm{k}}>0$ for all $\mathrm{k} \geqslant 0$.

Proof. If $u^{\sharp}$ solves SCFPP (1.2), then $u^{\sharp}=\mathcal{U} u^{\sharp}$ and $(\mathcal{J}-\mathcal{V}) \mathcal{A} u^{\sharp}=0$. It is obvious that $u^{\sharp} \in \operatorname{Fix}\left(\mathcal{U}-\mu_{n} \mathcal{A}^{*}(\mathcal{J}-\right.$ $\mathcal{V}) \mathcal{A}$ ) for any $\mu_{\mathrm{k}}>0$.

Conversely, setting $u^{\sharp} \in \operatorname{Fix}\left(\mathcal{U}-\mu_{k} \mathcal{A}^{*}(\mathcal{J}-\mathcal{V}) \mathcal{A}\right), \forall \mu_{k}>0$, we have

$$
\mathrm{u}^{\sharp}=\mathcal{U} \mathrm{u}^{\sharp}-\mu_{\mathrm{k}} \mathcal{A}^{*}(\mathcal{J}-\mathcal{V}) \mathcal{A} \mathrm{u}^{\sharp} \text {. }
$$

Picking up any $z \in \Omega$, we get

$$
\begin{aligned}
0 & =\left\langle u^{\sharp}-\mathcal{U} u^{\sharp}+\mu_{k} A^{*}(\mathcal{J}-\mathcal{V}) \mathcal{A} u^{\sharp}, u^{\sharp}-z\right\rangle \\
& =\left\langle u^{\sharp}-\mathcal{U} u^{\sharp}, u^{\sharp}-z\right\rangle+\mu_{k}\left\langle\mathcal{A}^{*}(\mathcal{J}-\mathcal{V}) \mathcal{A} u^{\sharp}, u^{\sharp}-z\right\rangle \\
& =\left\langle u^{\sharp}-\mathcal{U} u^{\sharp}, u^{\sharp}-z\right\rangle+\mu_{k}\left\langle(\mathcal{J}-\mathcal{V}) \mathcal{A} u^{\sharp}, \mathcal{A} u^{\sharp}-\mathcal{A} z\right\rangle .
\end{aligned}
$$

Since $\mathcal{U}$ and $\mathcal{V}$ are directed operator, from (2.1), we deduce

$$
\left\langle u^{\sharp}-\mathcal{u} u^{\sharp}, u^{\sharp}-z\right\rangle \geqslant\left\|u^{\sharp}-\mathcal{u} u^{\sharp}\right\|^{2},
$$

and

$$
\left\langle(\mathcal{J}-\mathcal{V}) \mathcal{A} u^{\sharp}, \mathcal{A} u^{\sharp}-\mathcal{A} z\right\rangle \geqslant\left\|(\mathcal{J}-\mathcal{V}) \mathcal{A} u^{\sharp}\right\|^{2} .
$$

Hence,

$$
0=\left\langle u^{\sharp}-U u^{\sharp}+\mu_{k} \mathcal{A}^{*}(\mathcal{J}-\mathcal{V}) \mathcal{A} u^{\sharp}, u^{\sharp}-z\right\rangle \geqslant\left\|u^{\sharp}-U u^{\sharp}\right\|^{2}+\mu_{k}\left\|(\mathcal{J}-\mathcal{V}) \mathcal{A} u^{\sharp}\right\|^{2} .
$$

Thus, $u^{\sharp} \in \operatorname{Fix}(\mathcal{U})$ and $\mathcal{A} u^{\sharp} \in \operatorname{Fix}(\mathcal{V})$. That is, $u^{\sharp}$ solves SCFPP (1.2). The proof is completed. 
Applying Lemma 3.1, we present the following algorithm.

Algorithm 3.2. Initialization: let $x^{0}$ be arbitrary.

Iterative step: for $k \geqslant 0$, given the current iterate $x^{k}$ calculates the next iterate $x^{k+1}$ by the following Mann's form

$$
x^{k+1}=\left(1-\alpha_{k}\right) x^{k}+\alpha_{k}\left[\mathcal{U} x^{k}-\mu_{k} \mathcal{A}^{*}(\mathcal{J}-\mathcal{V}) \mathcal{A} x^{k}\right], k \geqslant 0,
$$

where $\left\{\alpha_{k}\right\}$ and $\left\{\mu_{k}\right\}$ are two sequences in $(0,1)$.

Theorem 3.3. Suppose $\mathcal{J}-U$ and $\mathcal{J}-\mathcal{V}$ are demiclosed at zero. Assume the following conditions are satisfied:

(i) $0<\liminf _{k \rightarrow \infty} \alpha_{k} \leqslant \lim \sup _{k \rightarrow \infty} \alpha_{k}<1$;

(ii) $\mu_{k} \in\left(0, \frac{1}{\alpha_{k}\|\mathcal{A}\|^{2}}\right)$.

Then the sequence $\left\{x^{k}\right\}$ generated by (3.1) converges weakly to a solution $u^{\sharp}$.

Proof. We first show that the sequence $\left\{x^{k}\right\}$ is Fejér-monotone with respect to $\Omega$. Picking up any $z \in \Omega$, from (2.1), we have

$$
\begin{aligned}
\left\langle x^{k}-\mathcal{U} x^{k}+\mu_{k} \mathcal{A}^{*}(\mathcal{J}-\mathcal{V}) \mathcal{A} x^{k}, x^{k}-z\right\rangle & =\left\langle x^{k}-U x^{k}, x^{k}-z\right\rangle+\mu_{k}\left\langle\mathcal{A}^{*}(\mathcal{J}-\mathcal{V}) \mathcal{A} x^{k}, x^{k}-z\right\rangle \\
& =\left\langle x^{k}-U x^{k}, x^{k}-z\right\rangle+\mu_{k}\left\langle(\mathcal{J}-\mathcal{V}) \mathcal{A} x^{k}, \mathcal{A} x^{k}-\mathcal{A} z\right\rangle \\
& \geqslant\left\|x^{k}-U x^{k}\right\|^{2}+\mu_{k}\left\|(\mathcal{J}-\mathcal{V}) \mathcal{A} x^{k}\right\|^{2} .
\end{aligned}
$$

By (3.1) and (2.2), we get

$$
\begin{aligned}
\left\|x^{k+1}-z\right\|^{2}= & \|\left(1-\alpha_{k}\right) x^{k}+\alpha_{k}\left[U\left(x^{k}-\mu_{k} \mathcal{A}^{*}(I-\mathcal{V}) \mathcal{A} x^{k}\right]-z \|^{2}\right. \\
= & \left\|x^{k}-z-\alpha_{k}\left[x^{k}-U x^{k}+\mu_{k} \mathcal{A}^{*}(\mathcal{J}-\mathcal{V}) \mathcal{A} x^{k}\right]\right\|^{2} \\
= & \left\|x^{k}-z\right\|^{2}-2 \alpha_{k}\left\langle x^{k}-U x^{k}+\mu_{k} A^{*}(\mathcal{J}-\mathcal{V}) \mathcal{A} x^{k}, x^{k}-z\right\rangle \\
& +\alpha_{k}^{2}\left\|x^{k}-U x^{k}+\mu_{k} \mathcal{A}^{*}(\mathcal{J}-\mathcal{V}) \mathcal{A} x^{k}\right\|^{2} \\
\leqslant & \left\|x^{k}-z\right\|^{2}-2 \alpha_{k}\left\langle x^{k}-U x^{k}+\mu_{k} \mathcal{A}^{*}(\mathcal{J}-\mathcal{V}) \mathcal{A} x^{k}, x^{k}-z\right\rangle \\
& +\alpha_{k}^{2}\left[\left\|x^{k}-U x^{k}\right\|+\left\|\mu_{k} \mathcal{A}^{*}(\mathcal{J}-\mathcal{V}) \mathcal{A} x^{k}\right\|\right]^{2} \\
\leqslant & \left\|x^{k}-z\right\|^{2}-2 \alpha_{k}\left\langle x^{k}-U x^{k}+\mu_{k} \mathcal{A}^{*}(\mathcal{J}-\mathcal{V}) \mathcal{A} x^{k}, x^{k}-z\right\rangle \\
& +\alpha_{k}^{2}\left[2\left\|x^{k}-U x^{k}\right\|^{2}+2 \mu_{k}^{2}\|\mathcal{A}\|^{2}\left\|(\mathcal{J}-\mathcal{V}) \mathcal{A} x^{k}\right\|^{2}\right] .
\end{aligned}
$$

This together with (3.2) implies that

$$
\begin{aligned}
\left\|x^{k+1}-z\right\|^{2} \leqslant & \left\|x^{k}-z\right\|^{2}-2 \alpha_{k}\left[\left\|x^{k}-\mathcal{U} x^{k}\right\|^{2}+\mu_{k}\left\|(\mathcal{J}-\mathcal{V}) \mathcal{A} x^{k}\right\|^{2}\right] \\
& +\alpha_{k}^{2}\left[2\left\|x^{k}-\mathcal{U} x^{k}\right\|^{2}+2 \mu_{k}^{2}\|\mathcal{A}\|^{2}\left\|(\mathcal{J}-\mathcal{V}) \mathcal{A} x^{k}\right\|^{2}\right] \\
= & \left\|x^{k}-z\right\|^{2}-2 \alpha_{k}\left(1-\alpha_{k}\right)\left\|x^{k}-U x^{k}\right\|^{2}-2 \alpha_{k} \mu_{k}\left(1-\alpha_{k} \mu_{k}\|\mathcal{A}\|^{2}\right)\left\|(\mathcal{J}-\mathcal{V}) \mathcal{A} x^{k}\right\|^{2} .
\end{aligned}
$$

By virtue of (3.3), we deduce that the sequence $\left\{x^{k}\right\}$ is Fejér-monotone due to $\alpha_{k} \in(0,1)$ and $\mu_{k} \in$ $\left(0, \frac{1}{\alpha_{k}\|\mathcal{A}\|^{2}}\right)$. Hence, the sequence $\left\|x^{k}-z\right\|$ is convergent.

Next, we show that every weak cluster point of the sequence $\left\{x^{k}\right\}$ belongs to the solution set of problem (1.2), i.e., $\omega_{w}\left(x^{k}\right) \subset \Omega$.

From (3.3) and the conditions (i) and (ii), we obtain that the sequence $\left\{\left\|x^{k}-z\right\|\right\}$ is monotonically decreasing and thus converges to some positive real $\lim l(z)$. Therefore,

$$
\lim _{\mathrm{k} \rightarrow \infty}\left\|(\mathcal{J}-\mathcal{V}) \mathcal{A} x^{\mathrm{k}}\right\|=0 \text {. }
$$

From the Fejér-monotonicity of $\left\{x^{k}\right\}$ it follows that the sequence is bounded. Denoting by $\tilde{x}$ a weak-cluster point of $\left\{x^{k}\right\}$, let $i=0,1,2, \cdots$ be the sequence of indices, such that

$$
w-\lim _{i \rightarrow \infty} x^{k_{i}}=\tilde{x}
$$


Then from (3.4) and closedness of $\mathcal{J}-\mathcal{V}$ at 0 we obtain

$$
\mathcal{V}(\mathcal{A} \tilde{x})=\mathcal{A} \tilde{x}
$$

from which it follows that $\mathcal{A} \tilde{x} \in \operatorname{Fix}(\mathcal{V})$.

Again from (3.3) and the convergence of the sequence $\left\{\left\|x^{k}-z\right\|\right\}$ we also have

$$
\lim _{k \rightarrow \infty}\left\|x^{k}-\mathcal{U} x^{k}\right\|=0
$$

which combined with the demiclosedness of $\mathcal{J}-\mathcal{U}$ at 0 and the weak convergence of $\left\{x^{k_{i}}\right\}$ to $\tilde{x}$ yields

$$
U(\tilde{x})=\tilde{x} .
$$

Hence, $\tilde{x} \in \operatorname{Fix}(\mathcal{U})$. Therefore, $\tilde{x} \in \Omega$.

To this end, all conditions of the well-known Opial's lemma 2.1 are all fulfilled. Consequently, $\chi^{k} \rightarrow \tilde{\chi}$. The proof is completed.

We can apply our results to SFP (1.3).

Algorithm 3.4. Initialization: let $\chi^{0}$ be arbitrary.

Iterative step: for $k \geqslant 0$, given the current iterate $x^{k}$ calculates the next iterate $x^{k+1}$ by the following Mann's form

$$
x^{k+1}=\left(1-\alpha_{k}\right) x^{k}+\alpha_{k}\left[\operatorname{proj}_{\mathfrak{e}} x^{k}-\mu_{k} \mathcal{A}^{*}\left(\mathcal{J}-\operatorname{proj}_{Q}\right) \mathcal{A} x^{k}\right], k \geqslant 0,
$$

where $\alpha_{k} \in(0,1)$ is a constant.

Theorem 3.5. If $0<\liminf _{k \rightarrow \infty} \alpha_{k} \leqslant \limsup _{k \rightarrow \infty} \alpha_{k}<1$ and $\mu_{k} \in\left(0, \frac{1}{\alpha_{k}\|\mathcal{A}\|^{2}}\right)$, then the sequence $\left\{x^{k}\right\}$ generated by (3.5) converges weakly to a solution of SFP (1.3).

\section{Acknowledgment}

This research is supported by the Natural Science Foundation of China (no. 11401388) and the Natural Science Foundation of Zhejiang (no. LQ13A010007, LY14A010006), the Natural Science Foundation of Jiangsu (no. 16KJB110016).

\section{References}

[1] H. H. Bauschke, J. M. Borwein, On projection algorithms for solving convex feasibility problems, SIAM Rev., 38 (1996), 367-426. 2

[2] H. H. Bauschke, P. L. Combettes, A weak-to-strong convergence principle for Fejér-monotone methods in Hilbert spaces, Math. Oper. Res., 26 (2001), 248-264. 2

[3] F. E. Browder, W. V. Petryshyn, Construction of fixed points of nonlinear mappings in Hilbert space, J. Math. Anal. Appl., 20 (1967), 197-228. 1

[4] C. Byrne, A unified treatment of some iterative algorithms in signal processing and image reconstruction, Inverse Problems, 20 (2004), 103-120. 1

[5] Y. Censor, T. Elfving, A multiprojection algorithm using Bregman projections in a product space, Numer. Algorithms, 8 (1994), 221-239. 1

[6] Y. Censor, A. Segal, The split common fixed point problem for directed operators, J. Convex Anal., 16 (2009), 587-600. 1

[7] S. S. Chang, L. Wang, Y. K. Tang, L. Yang, The split common fixed point problem for total asymptotically strictly pseudocontractive mappings, J. Appl. Math., 2012 (2012), 13 pages. 1

[8] P. L. Combettes, Quasi-Fejérian analysis of some optimization algorithms, Inherently parallel algorithms in feasibility and optimization and their applications, Haifa, (2000), 115-152, Stud. Comput. Math., North-Holland, Amsterdam, (2001). 2

[9] Z.-H. He, W.-S. Du, On hybrid split problem and its nonlinear algorithms, Fixed Point Theory Appl., 2013 (2013), 20 pages. 1

[10] T.-H. Kim, H.-K. Xu, Strong convergence of modified Mann iterations, Nonlinear Anal., 61 (2005), 51-60. 1 
[11] P. E. Maingé, Approximation methods for common fixed points of nonexpansive mappings in Hilbert spaces, J. Math. Anal. Appl., 325 (2007), 469-479. 1

[12] A. Moudafi, The split common fixed-point problem for demicontractive mappings, Inverse Problems, 26 (2010), 6 pages. 1

[13] K. Nakajo, W. Takahashi, Strong convergence theorems for nonexpansive mappings and nonexpansive semigroups, J. Math. Anal. Appl., 279 (2003), 372-379. 1

[14] Z. Opial, Weak convergence of the sequence of successive approximations for nonexpansive mappings, Bull. Amer. Math. Soc., 73 (1967), 591-597. 2.1

[15] N. Shioji, W. Takahashi, Strong convergence of approximated sequences for nonexpansive mappings in Banach spaces, Proc. Amer. Math. Soc., 125 (1997), 3641-3645. 1

[16] T. Suzuki, Strong convergence of Krasnoselskii and Mann's type sequences for one-parameter nonexpansive semigroups without Bochner integrals, J. Math. Anal. Appl., 305 (2005), 227-239. 1

[17] F. Wang, Solving the split common fixed point problem by a fixed-point algorithm, J. Nonlinear Sci. Appl., (In press). 1

[18] F.-H. Wang, H.-K. Xu, Cyclic algorithms for split feasibility problems in Hilbert spaces, Nonlinear Anal., 74 (2011), 4105-4111. 1

[19] R. Wittmann, Approximation of fixed points of nonexpansive mappings, Arch. Math. (Basel), 58 (1992), 486-491. 1

[20] H.-K. Xu, Viscosity approximation methods for nonexpansive mappings, J. Math. Anal. Appl., 298 (2004), 279-291. 1

[21] H.-K. Xu, Iterative methods for the split feasibility problem in infinite-dimensional Hilbert spaces, Inverse Problems, 26 (2010), 17 pages. 1

[22] Y.-H. Yao, R.-D. Chen, J.-C. Yao, Strong convergence and certain control conditions for modified Mann iteration, Nonlinear Anal., 68 (2008), 1687-1693. 1

[23] Y.-H. Yao, L.-M. Leng, M. Postolache, X.-X. Zheng, Mann-type iteration method for solving the split common fixed point problem, J. Nonlinear Convex Anal., in press. 1

[24] Y.-H. Yao, Y.-C. Liou, T.-L. Lee, N.-C. Wong, An iterative algorithm based on the implicit midpoint rule for nonexpansive mappings, J. Nonlinear Convex Anal., 17 (2016), 655-668. 1

[25] Y.-H. Yao, N. Shahzad, Y.-C. Liou, Modified semi-implicit midpoint rule for nonexpansive mappings, Fixed Point Theory Appl., 2015 (2015), 15 pages. 1 